\title{
Microwave-assisted 1,3-dipolar cycloaddition reactions of vinylic glycosides with aryl azides - unexpected synthesis of triazoles and acetyl group migration
}

\author{
Marta M. Andrade* and Maria Teresa Barros* \\ REQUIMTE/CQFB, Departamento de Química, Faculdade de Ciências e Tecnologia, \\ Universidade Nova de Lisboa, 2829-516 Caparica, Portugal \\ E-mail: marta.andrade@,dq.fct.unl.pt, mtbarros@dq.fct.unl.pt
}

\begin{abstract}
The synthesis of sugar-derived triazolines was attempted under microwave irradiation, by 1,3dipolar cycloaddition to azides. However, due to the "push-pull" nature of the dipolarophile, we have obtained triazoles by elimination of the sugar unit, which then has one free hydroxyl group at a selective carbon position. The triazoles obtained are important precursors of anticonvulsive drugs. Furthermore, we have observed that under microwave irradiation of $300 \mathrm{~W}$ it is possible to promote acetyl group migration to a free hydroxyl group within the carbohydrate moiety.
\end{abstract}

Keywords: Microwave, 1,3-dipolar cycloaddition, azides, triazoles, acetyl group migration

\section{Introduction}

Azides are very important compounds due to their biological and industrial applications. ${ }^{1,2}$ One of their most useful synthetic applications is the preparation of 1,2,3-triaza compounds, via 1,3dipolar cycloaddition. ${ }^{3}$ Consequently, azides react with olefinic dipolarophiles to create $\Delta^{2}-1,2,3$ triazolines and with acetilenic dipolarophiles to originate 1,2,3-triazoles. While the latter are easily isolated, triazolines may have different stabilities depending on their substituents. Most of them are unstable and decompose by loosing nitrogen, affording products such as aziridines, diazo compounds, piperidines and pirrolidines. ${ }^{4}$

The chemistry of 1,2,3-triazoles acquired attention due to the diversity of their possible applications. In fact, they have been used as fungicides, herbicides, light stabilizers, fluorescent whiteners, optical brightening agents and corrosion retardants. ${ }^{1}$ The derivatives of triazole also possess a wide variety of activities such as antitumor, anti-inflammation, antimicrobial, antifungal, antithrombotic, antiplatelet and antiviral., ${ }^{2,5}$ Moreover, some 1,2,3-triazole derivatives linked to carbohydrate moieties have shown antimicrobial and cytostatic activities, among other impotant pharmacological applications. ${ }^{1,6}$ 
In recent years hundreds of different heterocyclic compounds have been synthesized and screened for anticonvulsant activity. ${ }^{7-9}$ These include mostly five and six membered ring systems containing up to three or four heteroatoms and seven membered ring systems related to the diazepines. Among the nitrogen containing heterocycles, a considerable amount of work has been done in the areas of five membered rings bearing one or two nitrogen atoms. Five membered rings with 3 or 4 nitrogen atoms that have been investigated include mostly, 1,2,4triazoles and some tetrazoles. However very little research has been done on 1,2,3-triazoles. ${ }^{10}$

One of the possible routes for the synthesis of 1,2,3-triazoles is based on the 1,3-dipolar cycloaddition reaction of organic azides and acetylenic compounds. Generally, two regioisomers can be obtained, leading to a low regioselectivity. An alternative approach consists in the regioselective reaction of azides to "push-pull" alkynes.

\section{Results and Discussion}

We have previously synthesized several glucose and sucrose derivatives appending a vinyl ethertype $\pi$ system at their primary carbon positions. ${ }^{11}$ With the view of obtaining new carbohydratederived 1,4-substituted triazolines, we have decided to explore their 1,3-dipolar cycloaddition reactions with benzilic azides.

The first attempt involved the use of a sucrose derivative (1) with a vinyl ester-type unsaturated chain appended at the most hindered primary carbon position of sucrose (C-1'), which reacted with $p$-methoxybenzylazide 2 under conventional heating conditions (Scheme 1).
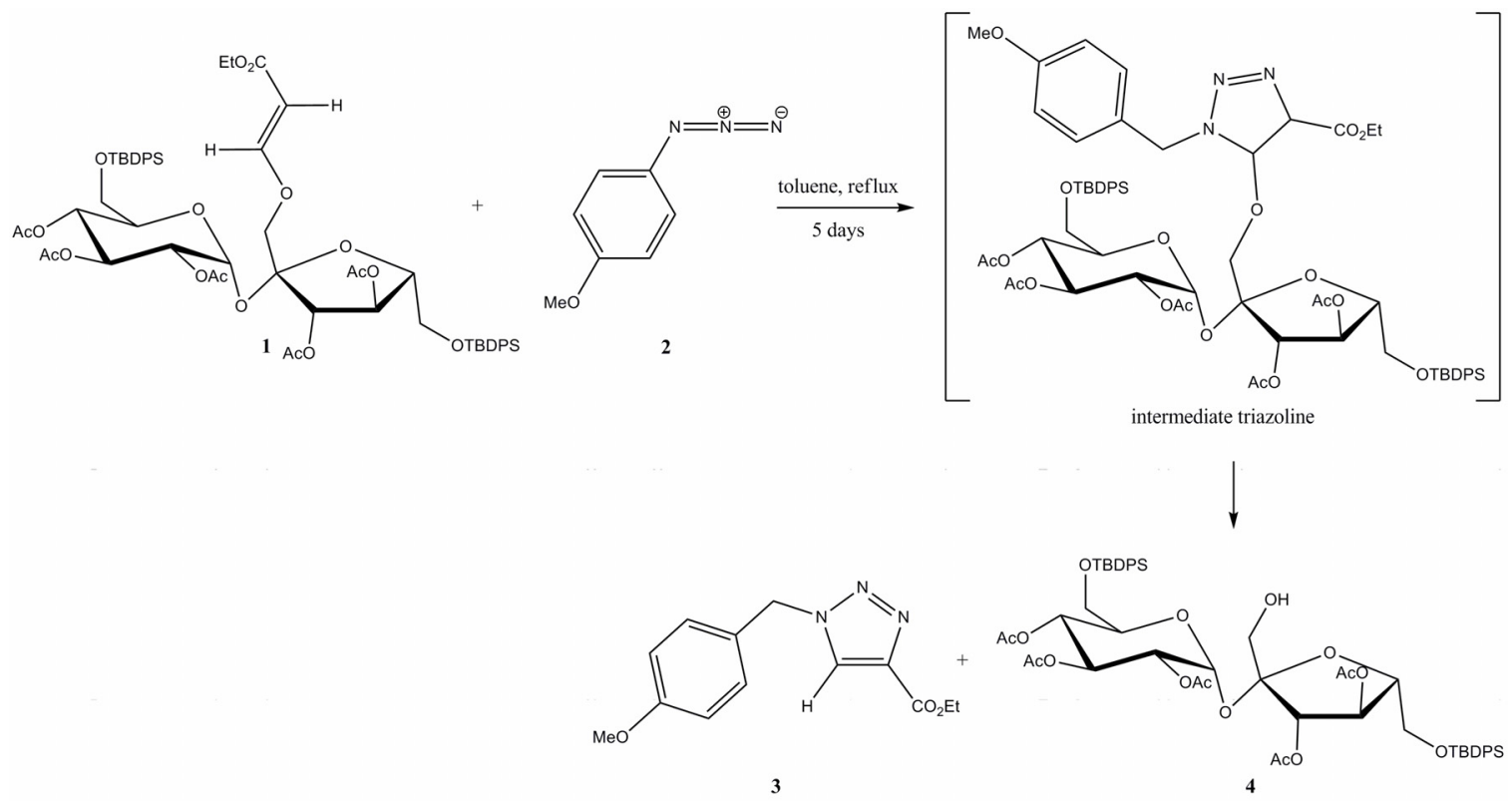

Scheme 1. 1,3-dipolar cycloaddition under conventional heating.

After 5 days refluxing we have obtained $76 \%$ of triazole $3,58 \%$ of sucrose derivative 4 and recovered $18 \%$ of starting sugar 1 . Due to the "push-pull" nature of the unsaturated side chain 
appended to the carbohydrate, instead of obtaining the sucrose-derived triazoline, the sugar moiety worked as leaving group, avoiding decomposition of the triazoline by loss of nitrogen and favoring the re-aromatization of the heterocyclic ring, leading to the corresponding 1,4substituted triazole (3). The reaction was monitored by TLC and we had never detected the presence of an intermediate triazoline. The sugar unit $\mathbf{4}$ has now one free hydroxyl group, which can be re-functionalized and so recycled for the same reaction or used for other proposes. The triazoline obtained is 1,4-disubstituted, without evidence of its regioisomer (1,5-disubstituted). This regioselectivity is induced by the presence of an electrondonating and leaving group - the sugar - and an electronwithdrawing group - the ester - in each side of the olefin, favoring the attack of the terminal nitrogen atom of the azide to the more nucleophilic carbon atom.

During the last decades, microwave radiation (MW) has gained the attention of chemists especially when combined with neat procedures. In the past 2 years, we have studied efficient strategies for the synthesis of unsaturated sugars using MW heating, ${ }^{11}$ developed potentially biodegradable polymers by copolymerisation with sugar monomers under MW irradiation ${ }^{12}$ and also applied MW-assisted neat procedures in the synthesis of nitrones and isoxazolidines. ${ }^{13}$

In an effort to improve the previous results from cycloaddition reaction, in shorter times and by use of cleaner processes, a microwave-assisted solventless protocol was exploited. Three different carbohydrate derivatives (1, 5 and 6) were chosen, which reacted with $p$ methoxybenzylazide (2) and benzylazide (7), under MW irradiation in the absence of solvent.

The reaction is schematized in Scheme 2 and the results obtained are summarized in Table 1.

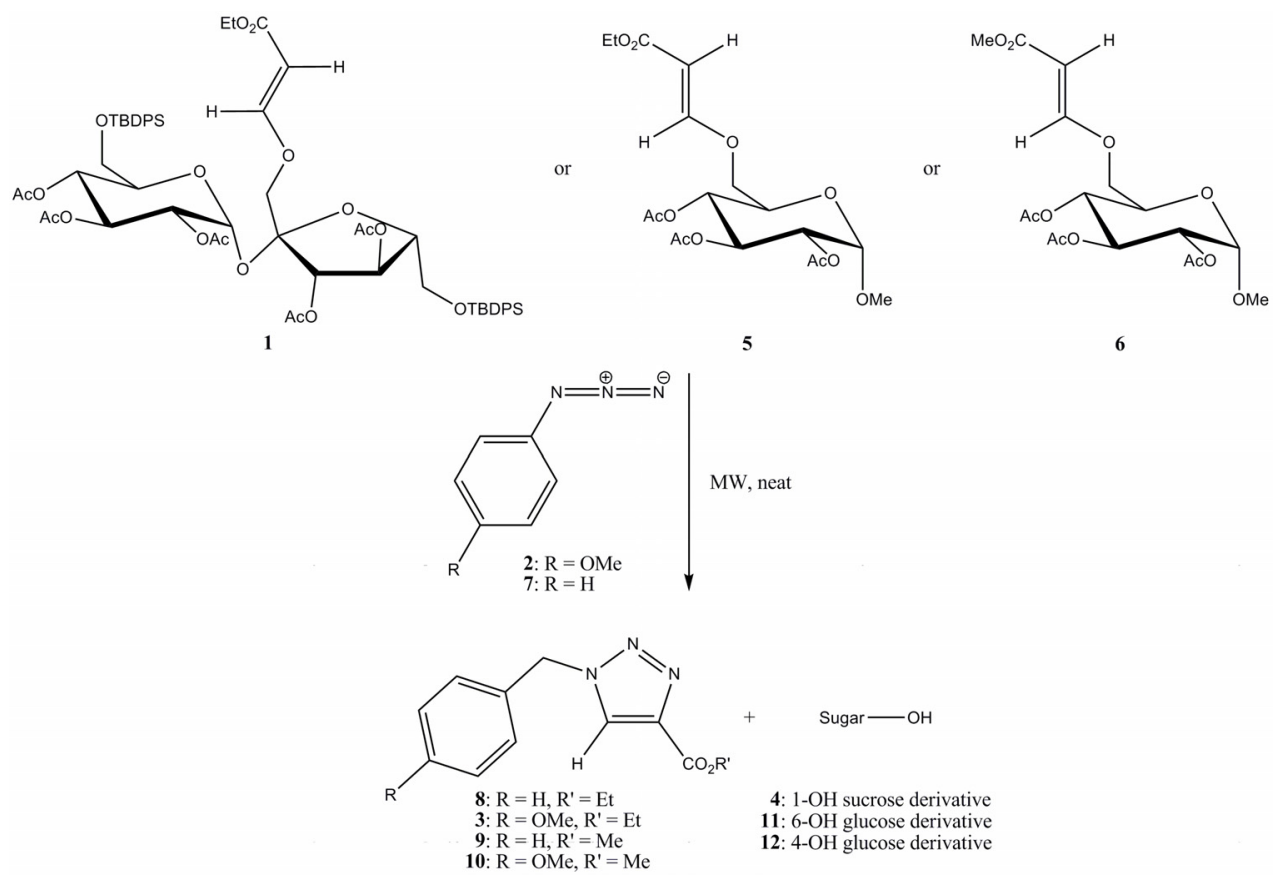

Scheme 2. MW promoted 1,3-dipolar cycloaddition. 
Likewise the results obtained with conventional heating, we have observed under microwave irradiation that the carbohydrate derivative plays two important roles in the cycloaddition reaction: it acts as an electrodonating group in a way that controls the regioselectivity of the reaction, but also as good leaving group, avoiding decomposition of the intermediate triazoline with formation of the corresponding triazole.

Table 1. Results obtained on microwave-assisted cycloaddition

\begin{tabular}{ccccc}
\hline Entry & Sugar & Azide & $\begin{array}{c}\text { Reaction condition } \\
\text { (Power/Time) }\end{array}$ & Results \\
\hline 1 & $\mathbf{1}$ & $\mathbf{2}$ & $300 \mathrm{~W} / 30 \mathrm{~min}$ & $\mathbf{4}($ quant $)+\mathbf{3}(87 \%)$ \\
2 & $\mathbf{5}$ & $\mathbf{7}$ & $300 \mathrm{~W} / 15 \mathrm{~min}$ & $\mathbf{1 1}(27 \%)+\mathbf{1 2}(22 \%)+\mathbf{8}(50 \%)$ \\
3 & $\mathbf{5}$ & $\mathbf{2}$ & $300 \mathrm{~W} / 15 \mathrm{~min}$ & $\mathbf{1 1}(17 \%)+\mathbf{1 2}(37 \%)+\mathbf{3}(74 \%)$ \\
4 & $\mathbf{5}$ & $\mathbf{2}$ & $200 \mathrm{~W} / 30 \mathrm{~min}$ & $\mathbf{1 1}(65 \%)+\mathbf{3}(55 \%)$ \\
5 & $\mathbf{6}$ & $\mathbf{2}$ & $100 \mathrm{~W} / 15 \mathrm{~min}$ & No reaction \\
6 & $\mathbf{6}$ & $\mathbf{2}$ & $200 \mathrm{~W} / 15 \mathrm{~min}$ & $\mathbf{1 1}(6 \%)+\mathbf{1 0}(23 \%)+\mathbf{6}($ recov. $61 \%)$ \\
7 & $\mathbf{6}$ & $\mathbf{7}$ & $100 \mathrm{~W} / 15 \mathrm{~min}$ & No reaction \\
8 & $\mathbf{6}$ & $\mathbf{7}$ & $200 \mathrm{~W} / 30 \mathrm{~min}$ & $\mathbf{1 1}(65 \%)+\mathbf{9}(70 \%)$ \\
9 & $\mathbf{6}$ & $\mathbf{7}$ & $300 \mathrm{~W} / 15 \mathrm{~min}$ & $\mathbf{1 1}(58 \%)+\mathbf{1 2}(6 \%)+\mathbf{9}(78 \%)$ \\
\hline
\end{tabular}

As predicted, the use of MW radiation drastically decreased reaction times and resulted in higher yields, due to complete consumption of the starting sugars. In entry 1, the results obtained for sucrose derivative at $300 \mathrm{~W}$ were much better than those using conventional heating and the conversion was complete in only 30 minutes. For glucose derivatives $\mathbf{5}$ and $\mathbf{6}$, different powers and times were screened. The use of $100 \mathrm{~W}$ power for glucose derivative 6 (entries 5 and 7) did not result in any cycloaddition products after $15 \mathrm{~min}$ of irradiation, neither by extending reaction time up to 1 hour (not indicated in the table). Increasing the power to $200 \mathrm{~W}$ promotes occurrence of reaction and after 15 min (entry 6) two products were obtained (sugar with free OH group 11, and triazole 10), but more than half of the starting sugar (6) was recovered. By extending the reaction time up to 30 min (entries 4 and 8), the conversion was total and the yields obtained were good. However, when increasing the power to $300 \mathrm{~W}$ (entries 2, 3 and 9), after only $15 \mathrm{~min}$ we have observed acetyl group migration from carbon position 4 to C-6 of the glucose derivatives.

Acyl group migrations are described in the literature by several methods and promoted by several reagents ${ }^{14-19}$ or enzymes. ${ }^{20}$ An acyl group may migrate to a neighboring $\mathrm{OH}$ under acidic and neutral, but especially under basic conditions. However we did not find any study in the literature on microwave-promoted migration. Our results show that such migration can be achieved by use of microwave irradiation at $300 \mathrm{~W}$ power, in short reaction times. 
The triazoles obtained $(\mathbf{3}, \mathbf{8}-\mathbf{1 0})$ in the present essay can be easily converted in 1-substituted benzyl- $N$-substituted-1,2,3-triazole-4-formamides by reaction with an amine and this family of compounds have recently shown antiepileptic activity. ${ }^{5}$

In conclusion, by use of neat microwave synthesis we have synthesized useful triazoles in good yield, which are precursors of antiepileptic drugs, leaving the sugar with free $\mathrm{OH}$ group ready for new approaches. Secondly, we have shown that acetyl group migration can be easily promoted under microwave irradiation in short reaction times.

\section{Experimental Section}

General. All solvents were purified before use. All reactions were monitored by thin layer chromatography, which was performed on aluminium-backed silica gel Merck $60 \mathrm{~F}_{254}$ plates, and compounds were detected by ultraviolet light or by staining with $10 \%$ solution of $\mathrm{H}_{2} \mathrm{SO}_{4}$ in ethanol, followed by heating. Flash chromatography was carried out using silica gel from Macherey-Nagel (Kieselgel $60 \mathrm{M}$ ). Preparative layer chromatography was performed on glass plates coated with $1 \mathrm{~mm}$ of silica gel (Mackerey-Nagel, Kieselgel $\mathrm{DGF}_{254}$ ). Elemental analyses were performed on Thermo Finnigan-CE Flash EA 1112 CHNS series analyzer. NMR spectra were recorded on a Bruker AMX-400MHz apparatus in $\mathrm{CDCl}_{3}$, using TMS as internal standard, with chemical shift values $(\delta)$ in ppm. Structural assignment of all new compounds was made by bi-dimensional NMR techniques (COSY 45, HMQC, NOESY, TOCSY). Microwave experiments were conducted in a MilestoneMicroSYNTH apparatus. Temperatures were measured at the surface of the vessel with a built-in contactless infrared sensor which provides a $\pm 1^{\circ} \mathrm{C}$ precision (according to manufacturer)

\section{Procedure for cycloaddition following conventional heating techniques}

A solution containing $100 \mathrm{mg}(0.09 \mathrm{mmol})$ of $\mathbf{3}$ and $28.9 \mathrm{mg}$ (2 equiv., $0.18 \mathrm{mmol})$ of 2 in $5 \mathrm{~mL}$ of toluene was refluxed for 5 days. The crude was purified by flash chromatography in ethyl ether /n-hexane $1 / 1$.

\section{General procedure for microwave-assisted cycloadditions}

$100 \mathrm{mg}$ of sugar derivative and $0.2 \mathrm{~mL}$ of azide were placed in an open flask, which was submitted to microwave irradiation in a Milestone Mycrosynth Labstation, with the power and times indicated in the table. The products were separated by flash chromatography.

\section{Selected spectroscopic data}

Ethyl 1-(4-methoxybenzyl)-1H-1,2,3-triazole-4-carboxylate (3). Mp 94-95 $\mathrm{C}$ (mp lit. ${ }^{21}$ 99$\left.\left.100^{\circ} \mathrm{C}\right) ;{ }^{1} \mathrm{H} \mathrm{NMR}\left(\mathrm{CDCl}_{3}\right) \delta 7.93\left(\mathrm{~s}, 1 \mathrm{H}, H-\mathrm{C}=\mathrm{C}-\mathrm{CO}_{2} \mathrm{Et}\right)\right), 7.25\left(\mathrm{~d}, 2 \mathrm{H}, J=8.4, \mathrm{Ph}-H_{\text {orto }}\right), 6.92$ (d, $2 \mathrm{H}, J=8.3$, Ph- $\left.H_{\text {meta }}\right), 5.51$ (s, $\left.2 \mathrm{H}, \mathrm{Ph}-\mathrm{CH}_{2}-\mathrm{N}\right), 4.40$ (q, $\left.2 \mathrm{H}, J=7.0, \mathrm{CH}_{2}-\mathrm{CH}_{3}\right), 3.82(\mathrm{~s}, 3 \mathrm{H}, \mathrm{Ph}-$ $\left.\mathrm{OCH}_{3}\right), 1.38\left(\mathrm{t}, 3 \mathrm{H}, J=7.1, \mathrm{CH}_{2}-\mathrm{CH}_{3}\right) \mathrm{ppm} .{ }^{13} \mathrm{C} \mathrm{NMR}\left(\mathrm{CDCl}_{3}\right) \delta 160.7(\mathrm{~s}, \mathrm{C}=\mathrm{O}), 160.2$ (s, MeO- 
$C(\mathrm{Ph})), 140.5\left(\mathrm{~d}, \mathrm{EtO}_{2} \mathrm{C}-\mathrm{C}=\mathrm{C}-\mathrm{H}\right), 129.6\left(\mathrm{~d}, C\right.$ - $\left.\mathrm{H}_{\text {orto }}(\mathrm{Ph})\right), 127.0\left(\mathrm{~d}, \mathrm{EtO}_{2} \mathrm{C}-\mathrm{C}=C-\mathrm{H}\right), 125.6$ (s, $\left.\mathrm{CH}_{2}-\mathrm{C}(\mathrm{Ph})\right), 114.7$ (d, $\left.C-\mathrm{H}_{\text {meta }}(\mathrm{Ph})\right), 61.2\left(\mathrm{t}, \mathrm{CH}_{2}-\mathrm{CH}_{3}\right), 55.3$ (q, $\left.\mathrm{Ph}-\mathrm{OCH}_{3}\right), 54.0\left(\mathrm{t}, \mathrm{N}-\mathrm{CH}_{2}-\mathrm{Ph}\right)$, 14.3 (q, $\mathrm{CH}_{2}-\mathrm{CH}_{3}$ ) ppm. F.T.-I.R. ( $\mathrm{NaCl}$ ): 1730 (vs, $\left.\mathrm{C}=\mathrm{O}\right), 1613$ (vs), 1516 (s) cm $\mathrm{cm}^{-1}$.

$\mathbf{2 , 3 , 3}, \mathbf{4 , 4} \mathbf{4}^{\prime}$-Penta- $\boldsymbol{O}$-acetyl-6,6'-di- $\boldsymbol{O}$-TBDPS-sucrose (4). $[\alpha]_{\mathrm{D}}^{20}=+46.8$ (c $\left.0.5, \mathrm{CHCl}_{3}\right)$. ${ }^{1} \mathrm{H}$ NMR $\left(\mathrm{CDCl}_{3}\right) \delta 7.60\left(\mathrm{~m}, 8 \mathrm{H}, \mathrm{Ph}-H_{\text {orto }}\right), 7.33\left(\mathrm{~m}, 12 \mathrm{H}, \mathrm{Ph}-H_{\text {meta, para }}\right), 5.61$ (d, $\left.1 \mathrm{H}, J=3.6, \mathrm{H}-1\right) 5.55(\mathrm{t}$, $1 \mathrm{H}, J=5.1, \mathrm{H}-4$ ') 5.38 (t, 1H, $J=9.9, \mathrm{H}-3), 5.32$ (d, 1H, $\left.J=5.2, \mathrm{H}-3^{\prime}\right), 5.22$ (t, 1H, $J=9.8, \mathrm{H}-4$ ), 4.88 (dd, 1H, $\left.J_{1,2}=3.6, J_{2,3}=10.4, \mathrm{H}-2\right), 4.09$ (q, $1 \mathrm{H}, J=5.5, \mathrm{H}-5$ '), 3.98 (bdd, $1 \mathrm{H}, J_{5,6}=1.5, J_{4,5}=10$, H-5), 3.83 (dd, $\left.1 \mathrm{H}, J_{5}{ }^{\prime}, 6^{\prime} \alpha=5.8, J_{6}{ }^{\prime} \alpha,{ }^{\prime}{ }^{\beta}=10.6, \mathrm{H}-6{ }^{\prime} \alpha\right), 3.79$ (d, $1 \mathrm{H}, J_{6}{ }^{\prime} \alpha, 6^{\prime}{ }^{\prime}=10.4, \mathrm{H}-6$ ' $\beta$ ), 3.73 (dd, $\left.1 \mathrm{H}, J_{\mathrm{OH}, 1^{\prime} \beta}=7.7, J_{1^{\prime} \alpha, 1^{\prime} \beta}=12.6, \mathrm{H}-1^{\prime} \beta\right), 3.64\left(\mathrm{dd}, 1 \mathrm{H}, J_{\mathrm{OH}, 1^{\prime} \alpha}=4.4, J_{1^{\prime} \alpha, 1^{\prime} \beta}=12.3, \mathrm{H}-1^{\prime} \alpha\right), 3.53(\mathrm{~d}, 1 \mathrm{H}$, $\left.J_{6 \alpha, 6 \beta}=10.4, \mathrm{H}-6 \alpha\right), 3.44\left(\mathrm{dd}, 1 \mathrm{H}, J_{5,6 \beta}=3.8, J_{6 \alpha, 6 \beta}=11.5, \mathrm{H}-6 \beta\right), 2.63(\mathrm{t}, 1 \mathrm{H}, J=5.1, \mathrm{OH}), 2.13,2.10$, 2.01, $1.85\left(4 \mathrm{~s}, 15 \mathrm{H}, 5 \mathrm{CH}_{3}\right), 1.01,1.01\left(2 \mathrm{~s}, 18 \mathrm{H}, 2^{t} \mathrm{Bu}-H\right) \mathrm{ppm} .{ }^{13} \mathrm{C} \mathrm{NMR}\left(\mathrm{CDCl}_{3}\right) \delta 170.6,170.2$, 170.1, 169.7, 169.2 (s, $C=\mathrm{O}(\mathrm{Ac})), 135.6,135.4\left(\mathrm{~d}, C-\mathrm{H}_{\text {orto }}(\mathrm{Ph})\right), 133.0,132.7(\mathrm{~s}, C(\mathrm{Ph})), 129.7$, 129.6 (d, $\left.C-\mathrm{H}_{\text {para }}(\mathrm{Ph})\right), 127.7,127.6$ (d, C-H $\left.\mathrm{H}_{\text {meta }}(\mathrm{Ph})\right), 105.8$ (s, C-2'), 90.6 (d, C-1), 81.3 (d, C5'), 77.9 (d, C-3'), 76.4 (d, C-4'), 70.5 (d, C-5), 70.3 (d, C-2), 70.1 (d, C-3), 68.2 (d, C-4), 64.3 (t, C-6'), 63.2 (t, C-1'), 61.6 (t, C-6), 26.7, 26.7 (q, $\mathrm{CH}_{3}\left({ }^{t} \mathrm{Bu}\right)$ ), 20.8, 20.7, 20.6, 20.5 (q, $\mathrm{CH}_{3}$ (Ac)), 19.2, 19.1 (s, $\left.C\left({ }^{t} \mathrm{Bu}\right)\right)$ ppm. Anal. Calcd for $\mathrm{C}_{54} \mathrm{H}_{68} \mathrm{O}_{16} \mathrm{Si}_{2}$ : C, 63.01; H, 6.66. Found: C, 63.14 ; H 6.98.

Ethyl 1-benzyl-1H-1,2,3-triazole-4-carboxylate (8). Mp $89^{\circ} \mathrm{C}\left(\mathrm{mp} \mathrm{lit} .{ }^{22} 88-89^{\circ} \mathrm{C}\right)$; ${ }^{1} \mathrm{H}$ NMR $\left.\left(\mathrm{CDCl}_{3}\right) \delta 7.97\left(\mathrm{~s}, 1 \mathrm{H}, H-\mathrm{C}=\mathrm{C}-\mathrm{CO}_{2} \mathrm{Et}\right)\right), 7.39$ (m, 3H, Ph- $\left.H_{\text {orto,para }}\right), 7.29$ (m, 2H, Ph- $\left.H_{\text {meta }}\right), 5.58$ (s, 2H, Ph-CH $\left.H_{2}-\mathrm{N}\right), 4.40\left(\mathrm{q}, 2 \mathrm{H}, J=7.1, \mathrm{CH}_{2}-\mathrm{CH}_{3}\right), 1.38$ (t, 3H, $\left.J=7.1, \mathrm{CH}_{2}-\mathrm{CH}_{3}\right)$ ppm. ${ }^{13} \mathrm{C}$ NMR $\left(\mathrm{CDCl}_{3}\right) \delta 160.7(\mathrm{~s}, C=\mathrm{O}), 140.7\left(\mathrm{~d}, \mathrm{EtO}_{2} \mathrm{C}-C=\mathrm{C}-\mathrm{H}\right), 133.7\left(C-\mathrm{H}_{\text {para }}(\mathrm{Ph})\right), 129.3\left(C-\mathrm{H}_{\text {orto }}(\mathrm{Ph})\right)$, $129.2\left(\mathrm{EtO}_{2} \mathrm{C}-\mathrm{C}=C-\mathrm{H}\right), 128.3\left(C-\mathrm{H}_{\text {meta }}(\mathrm{Ph})\right), 127.2\left(\mathrm{CH}_{2}-\mathrm{C}(\mathrm{Ph})\right), 61.3\left(\mathrm{CH}_{2}-\mathrm{CH}_{3}\right), 54.5\left(\mathrm{~N}-\mathrm{CH}_{2}-\right.$ $\mathrm{Ph}), 14.3\left(\mathrm{CH}_{2}-\mathrm{CH}_{3}\right)$ ppm. F.T.-I.R. $(\mathrm{NaCl}): 1730$ (vs, $\left.\mathrm{C}=\mathrm{O}\right), 1542(\mathrm{~s}) \mathrm{cm}^{-1}$.

Methyl 1-benzyl-1H-1,2,3-triazole-4-carboxylate (9). Mp 103-104 ${ }^{\circ} \mathrm{C}$ (mp lit. ${ }^{23} 104^{\circ} \mathrm{C}$ ); ${ }^{1} \mathrm{H}$ $\left.\operatorname{NMR}\left(\mathrm{CDCl}_{3}\right) \delta 7.98\left(\mathrm{~s}, 1 \mathrm{H}, H-\mathrm{C}=\mathrm{C}-\mathrm{CO}_{2} \mathrm{Et}\right)\right), 7.39$ (m, 3H, Ph- $\left.H_{\text {orto,para }}\right), 7.30$ (m, 2H, Ph- $\left.H_{\text {meta }}\right)$, $5.58\left(\mathrm{~s}, 2 \mathrm{H}, \mathrm{Ph}-\mathrm{CH}_{2}-\mathrm{N}\right), 3.93\left(\mathrm{~s}, 3 \mathrm{H}, \mathrm{O}=\mathrm{C}-\mathrm{OCH}_{3}\right)$ ppm. ${ }^{13} \mathrm{C} \mathrm{NMR}\left(\mathrm{CDCl}_{3}\right) \delta 161.0(\mathrm{~s}, \mathrm{C}=\mathrm{O})$, $140.3\left(\mathrm{~d}, \mathrm{MeO}_{2} \mathrm{C}-\mathrm{C}=\mathrm{C}-\mathrm{H}\right), 133.6\left(\mathrm{~d}, C-\mathrm{H}_{\text {para }}(\mathrm{Ph})\right), 129.3$ (d, $C$ - $\left.\mathrm{H}_{\text {orto }}(\mathrm{Ph})\right), 129.1$ (d, $\mathrm{MeO}_{2} \mathrm{C}-$ $\mathrm{C}=C-\mathrm{H}), 128.2\left(\mathrm{~d}, C-\mathrm{H}_{\text {meta }}(\mathrm{Ph})\right), 127.3\left(\mathrm{~s}, \mathrm{CH}_{2}-\mathrm{C}(\mathrm{Ph})\right), 54.5\left(\mathrm{t}, \mathrm{N}-\mathrm{CH}_{2}-\mathrm{Ph}\right), 52.12(\mathrm{q}, \mathrm{O}=\mathrm{C}-$ $\mathrm{OCH}_{3}$ ) ppm. F.T.-I.R. (NaCl): 1728 (vs, C=O), $1542(\mathrm{~s}), 1433(\mathrm{~m}) \mathrm{cm}^{-1}$.

Methyl 1-(4-methoxybenzyl)-1H-1,2,3-triazole-4-carboxylate (10). Mp 129-131 ${ }^{\circ} \mathrm{C}$ (mp lit. ${ }^{24}$ $\left.142-143{ }^{\circ} \mathrm{C}\right) ;{ }^{1} \mathrm{H} \mathrm{NMR}\left(\mathrm{CDCl}_{3}\right) \delta 7.94\left(\mathrm{~s}, 1 \mathrm{H}, H-\mathrm{C}=\mathrm{C}-\mathrm{CO}_{2} \mathrm{Me}\right), 7.25\left(\mathrm{~d}, 2 \mathrm{H}, J=8.5, \mathrm{Ph}-H_{\text {orto }}\right)$, $6.92\left(\mathrm{~d}, 2 \mathrm{H}, J=8.5\right.$, Ph- $\left.H_{\text {meta }}\right), 5.51$ (s, 2H, Ph-CH $\left.H_{2}-\mathrm{N}\right), 3.92$ (s, 3H, O=C-OCH$), 3.82$ (s, 3H, Ph$\left.\mathrm{OCH}_{3}\right)$ ppm. ${ }^{13} \mathrm{C}$ NMR $\left(\mathrm{CDCl}_{3}\right) \delta 161.1(\mathrm{~s}, \mathrm{C}=\mathrm{O}), 160.2(\mathrm{~s}, \mathrm{MeO}-\mathrm{C}(\mathrm{Ph})), 140.2\left(\mathrm{~d}, \mathrm{MeO}_{2} \mathrm{C}-\right.$ $C=\mathrm{C}-\mathrm{H}), 129.9\left(\mathrm{~d}, C-\mathrm{H}_{\text {orto }}(\mathrm{Ph})\right), 127.1\left(\mathrm{~d}, \mathrm{MeO}_{2} \mathrm{C}-\mathrm{C}=C-\mathrm{H}\right), 125.5\left(\mathrm{~s}, \mathrm{CH}_{2}-C(\mathrm{Ph})\right), 114.7(\mathrm{~d}, C-$ $\mathrm{H}_{\text {meta }}(\mathrm{Ph})$ ), 55.3 (q, Ph-OCH $H_{3}$, 54.1 (t, N-CH2 $\left.-\mathrm{Ph}\right), 52.14$ (q, O=C-OCH ) ppm. F.T.-I.R. (NaCl): 1722 (vs, C=O), $1612(\mathrm{~s}), 1515(\mathrm{~s}) \mathrm{cm}^{-1}$.

Methyl 2,3,4-tri- $\boldsymbol{O}$-acetyl- $\alpha$-D-glucopyranoside ${ }^{\mathbf{2 0}}$ (11). Mp $102-103^{\circ} \mathrm{C}\left(\mathrm{mp} \mathrm{lit.}{ }^{20} 103-104^{\circ} \mathrm{C}\right){ }^{1} \mathrm{H}$ $\operatorname{NMR}\left(\mathrm{CDCl}_{3}\right) \delta 5.54(\mathrm{t}, 1 \mathrm{H}, J=9.8, \mathrm{H}-3), 5.03(\mathrm{t}, 1 \mathrm{H}, J=9.8, \mathrm{H}-4), 4.97$ (d, 1H, $\left.J=3.5, \mathrm{H}-1\right), 4.88$ $\left(\mathrm{dd}, 1 \mathrm{H}, J_{1,2}=3.6, J_{2,3}=10.2, \mathrm{H}-2\right), 3.79\left(\mathrm{ddd}, 1 \mathrm{H}, J_{5,6 \alpha}=2.3, J_{5,6 \beta}=3.7, J_{4,5}=10, \mathrm{H}-5\right), 3.72(\mathrm{~d}, 1 \mathrm{H}$, $\left.J_{5,6 \alpha}=2.1, J_{6 \alpha, 6 \beta}=12.7, \mathrm{H}-6 \alpha\right), 3.60\left(\mathrm{dd}, 1 \mathrm{H}, J_{5,6 \beta}=3.9, J_{6 \alpha, 6 \beta}=12.6, \mathrm{H}-6 \beta\right), 3.42\left(\mathrm{~s}, 3 \mathrm{H}, \mathrm{OCH}_{3}\right), 2.34$ 
(bs, $1 \mathrm{H}, \mathrm{OH}), 2.08,2.07,2.02\left(3 \mathrm{~s}, 9 \mathrm{H}, 3 \mathrm{CH}_{3}(\mathrm{Ac})\right) \mathrm{ppm} .{ }^{13} \mathrm{C} \mathrm{NMR}\left(\mathrm{CDCl}_{3}\right) \delta 170.5,170.1,170.0$ (s, $C=$ ) ), 96.7 (d, C-1), 70.9 (d, C-2), 69.8 (d, C-3), 69.2 (d, C-5), 68.8 (d, C-4), 60.9 (t, C-6), 55.3 (q, $\mathrm{OCH}_{3}$ ), 20.6 (q, $\left.\mathrm{CH}_{3}(\mathrm{Ac})\right)$ ppm. F.T.-I.R. ( $\mathrm{NaCl}$ ): 3489 (br, O-H), 1749 (vs, $\mathrm{C}=\mathrm{O}(\mathrm{Ac})$ ), 1710 (vs, $\mathrm{C}=\mathrm{C}-\mathrm{C}=\mathrm{O}) \mathrm{cm}^{-1}$.

Methyl 2,3,6-tri- $\boldsymbol{O}$-acetyl- $\boldsymbol{\alpha}$-D-glucopyranoside ${ }^{\mathbf{2 0}}$ (12). ${ }^{1} \mathrm{H} \mathrm{NMR}\left(\mathrm{CDCl}_{3}\right) \delta 5.31$ (t, $1 \mathrm{H}, J=9.6$, $\mathrm{H}-3$ ), 4.91 (d, $1 \mathrm{H}, J=3.4, \mathrm{H}-1), 4.87$ (dd, $\left.1 \mathrm{H}, J_{1,2}=3.4, J_{2,3}=10.1, \mathrm{H}-2\right), 4.48$ (dd, $1 \mathrm{H}, J_{5,6 \beta}=4.2$, $\left.J_{6 \alpha, 6 \beta}=12.2, \mathrm{H}-6 \beta\right), 4.31$ (d, $\left.1 \mathrm{H}, J_{6 \alpha, 6 \beta}=12.1, \mathrm{H}-6 \alpha\right), 3.83$ (bd, $\left.1 \mathrm{H}, J_{4,5}=9.7, \mathrm{H}-5\right), 3.56(\mathrm{t}, 1 \mathrm{H}$, $J=8.9, \mathrm{H}-4), 3.41$ (s, 3H, OCH $\left.H_{3}\right), 3.05$ (bs, $\left.1 \mathrm{H}, \mathrm{OH}\right), 2.13,2.10,2.09$ (3s, 9H, 3CH $\left.\mathrm{CH}_{3}(\mathrm{Ac})\right) \mathrm{ppm}$.

${ }^{13} \mathrm{C}$ NMR $\left(\mathrm{CDCl}_{3}\right) \delta 171.6,171.5,170.3(\mathrm{~s}, \mathrm{C}=\mathrm{O}), 96.8(\mathrm{~d}, \mathrm{C}-1), 72.9(\mathrm{~d}, \mathrm{C}-3), 70.6(\mathrm{~d}, \mathrm{C}-2)$, 69.7 (d, C-5), 69.3 (d, C-4), 62.8 (t, C-6), 55.3 (q, $\mathrm{OCH}_{3}$ ), 20.7 (q, $\left.\mathrm{CH}_{3}(\mathrm{Ac})\right)$ ppm. F.T.-I.R. $(\mathrm{NaCl}): 3476(\mathrm{br}, \mathrm{O}-\mathrm{H}), 1744$ (vs, $\mathrm{C}=\mathrm{O}(\mathrm{Ac})) \mathrm{cm}^{-1}$.

\section{References}

1. Abu-Orabi, S. T. Molecules 2002, 7, 302.

2. Molteni, G.; Ponti, A. Arkivoc 2006, (xvi), 49.

3. Katritzky, A. R.; Zhang, Y.; Singh, S. K.; Steel, P. J. Arkivoc 2003, (xv), 47.

4. Markidis, T.; Mikros, E.; Kokotos, G. Heterocycles 2003 60, 2637.

5. Wang, J. M.; Jun, C.; Chai, K.; Kwak, K.; Quan, Z. S. Progr. Natur. Sci. 2006, 16, 925.

6. Wamhoff, H.; Warnecke, H. Arkivoc 2001, (ii), 95.

7. Malawska, B. Curr. Top. Med. Chem. 2005, 5, 69.

8. Zappalà, M.; Micale, N.; Grasso, S.; Menniti, F. S.; Sarro, G. D.; Micheli, C. D. Arkivoc 2004, (v), 196.

9. Ameta, U.; Ojha, S.; Bhambi, D.; Talesara, G. L. Arkivoc 2006, (xiii), 83.

10. Pankaja, K. K. J. Pharm. Sci. 1984, 73, 850.

11. Andrade, M. M.; Barros, M. T.; Rodrigues, P. Eur. J. Org. Chem. 2007, 3655.

12. Barros, M. T.; Petrova, K. T.; Ramos, A. M. Eur. J. Org. Chem. 2007, 1357.

13. Andrade, M. M.; Barros, M. T.; Pinto, R. C. Tetrahedron 2008, 64, 10521.

14. Graziani, A.; Passacantilli, P.; Piancatelli, G.; Tani, S. Tetrahedron Lett. 2001, 42, 3857.

15. Berdin, A. G.; Raldugin, V. A.; Shakirov, M. M.; Druganov, A. G.; Bagryanskaya, I. Y.; Gatilov, Y. V.; Kulyyasov, A. T.; Adekenov, S. M.; Tolstikov, G. A. Russ. Chem. Bull. 2001, 50, 2459.

16. Roslund, M. U.; Aitio, O.; Warna, J.; Maaheimo, H.; Murzin, D. Y.; Leino, R. J. Am. Chem. Soc. 2008, 130, 8769.

17. Zhang, J. J.; Liang, X. M.; Wang, D. Q.; Kong, F. Z. Carbohydr. Res. 2007, 342, 797.

18. Kroger, L.; Thiem, J. Carbohydr. Res. 2007, 342, (3-4), 467.

19. Chevallier, O. P.; Migaud, M. E. Beilstein J. Org. Chem. 2006, 2.

20. Horrobin, T.; Tran, C. H.; Crout, D. J. Chem. Soc., Perkin Trans. 1 1998, 1069. 
21. Sultan, T. A.-O.; Atfah, M. A.; Ibrahim, J.; Fakhri, M. M. i.; Amer Al-Sheikh, A. J. Heterocycl. Chem. 1989, 26, 1461.

22. Holzer, W. Tetrahedron 1991, 47, 9783.

23. Lübbe, F.; Grosz, K.-P.; Hillebrand, W.; Sucrow, W. Tetrahedron Lett. 1981, 22, 227.

24. Abu-Orabi, S. T.; Atfah, A.; Jibril, I.; Al-Sheikh Ali, A.; Marii, F. Gazz. Chim. Ital. 1992, $122,29$. 